Xavier GHEERBRANT, Empédocle : une poétique philosophique, (Kaïnon. Anthropologie de la pensée ancienne, 6), 931 p., ISBN : 978-2-406-05713-0

\title{
Anne-Laure Therme
}

\section{(2) OpenEdition}

1 Journals

\section{Édition électronique}

URL : https://journals.openedition.org/philosant/1073

DOI : $10.4000 /$ philosant.1073

ISSN : 2648-2789

Éditeur

Éditions Vrin

\section{Édition imprimée}

Date de publication : 1 novembre 2018

Pagination : 281-283

ISBN : 978-2-7574-2372-1

ISSN : 1634-4561

\section{Référence électronique}

Anne-Laure Therme, «Xavier GheErbrant, Empédocle : une poétique philosophique, (Kaïnon. Anthropologie de la pensée ancienne, 6), 931 p., ISBN : 978-2-406-05713-0 », Philosophie antique [En ligne], 18| 2018, mis en ligne le 01 novembre 2018, consulté le 03 décembre 2022. URL : http:// journals.openedition.org/philosant/1073; DOI : https://doi.org/10.4000/philosant.1073

\section{(c)}

Creative Commons - Attribution - Pas d'Utilisation Commerciale - Pas de Modification 4.0 International - CC BY-NC-ND 4.0

https://creativecommons.org/licenses/by-nc-nd/4.0/ 


\section{COMPTES RENDUS}

Xavier Gheerbrant, Empédocle : une poétique philosophique, Classiques Garnier, Paris, 2017 (Kaïnon. Anthropologie de la pensée ancienne, 6), 931 p., ISBN : 978-2-406-05713-0.

Cet ouvrage dense, issu d'une thèse de doctorat en langue et littérature grecques soutenue à Lille en 2014, vise à combler une lacune des études sur Empédocle en examinant la relation essentielle de sa philosophie à la forme versifiée : loin dêtre un vêtement ou un ornement archaïsant, le « véhicule poétique » de l'hexamètre dactylique épique était conçu par Empédocle comme la condition nécessaire de l'expression de sa pensée. La forme poétique est ici constitutive du contenu philosophique même, en tant non seulement quelle intègre dans le dispositif textuel des traits et indices doctrinaux, mais aussi s'inscrit dans une tradition qu'elle bouscule et refonde.

La méthodologie se place sous l'égide de Jean Bollack (dans l'intention d'en poursuivre l'œuvre et d’en fonder les présupposés, p. 721) et de l'herméneutique critique de l'École de Lille. Procédant d'abord à une analyse syntaxique et sémantique des fragments, mise en relation avec la tradition poétique antérieure, et prêtant une attention particulière à leurs sources et contextes dénonciation, l'auteur recense les différentes positions interprétatives et leurs enjeux, qu'il confronte alors aux résultats obtenus par l'analyse philologique pour en réfuter certains et formuler sa propre interprétation. Priorité est toujours donnée aux textes des manuscrits, que l'auteur s'efforce à raison de défendre contre les lectures qui, prétendant restaurer l'intelligibilité des textes, les corrigent ad hoc. Les fragments étudiés et leurs sources, traduits par l'auteur, sont donnés en Annexe avec leur apparat critique.

La première partie, « la théorie poétique » (p. 40-242) s’emploie à montrer pourquoi Empédocle écrit en vers en un temps où la prose s'impose comme dispositif textuel privilégié de l'expression philosophique, en se penchant plus spécifiquement sur la figure de la Muse qu'invoque le poète. L'auteur sattaque à la reconstruction de fragments difficiles, tant dans leur forme que dans les problèmes interprétatifs qu'ils soulèvent, en veillant à respecter la lettre des manuscrits. Les deux premiers chapitres montrent que le discrédit jeté sur les sens au fr. 2 (inscrit dans le dispositif textuel même) est conciliable avec le modèle de leur bon usage décrit au fr. 3 ; celui-ci présuppose la connaissance d'une vérité transcendante que seule la parole poétique, portée par la Muse, peut révéler. Le poème serait ainsi métapoétique : Empédocle aurait formulé dans ses vers une théorie poétique dont la compréhension est indispensable au disciple pour accéder à la vérité philosophique qu'ils renferment. « L'étude de la construction de la théorie de composition poétique que le poème se donne » est ainsi dite «plus fondamentale » que « la doctrine physiologique, physique et cosmologique » (p. 103). L'auteur montre par des analyses détaillées et nourries comment Empédocle construit la figure de sa Muse en rupture avec la tradition poétique épique et hymnique : caractérisée par la pureté de la parole quelle inspire, au moyen d'un réseau de métaphores et d'épithètes reliées à l'Amour, elle est opposée à la folie ( $\mu \alpha v i ́ n)$, d'habitude associée à la parole d'inspiration divine, mais ici aux discours trompeurs. Sous couvert d'une structure traditionnelle de discours sur les dieux, Empédocle en renverse le contenu (les vrais dieux ne sont pas ceux du Panthéon, mais l'Amour et les quatre éléments) par une subversion subtile de la forme et des connotations attendues, pour préparer l'auditeur à l'originalité du contenu, mais aussi pour s’opposer aux poètes théogoniques dont la Muse est dévoyée, et qui tissent des discours mensongers pour conquérir la gloire. La reconstruction de la fin du fr. 3 est emblématique de la manière dont l'auteur procède, donnant sens à 
la leçon des manuscrits (ici, à la syntaxe problématique du v. 9) : il faut comprendre qu'Empédocle enjoint au disciple, non pas de faire usage de chacun de ses sens ou « paumes » séparément, mais de percevoir par tout son corps, d'unifier ses sensations, ce qui exige de suivre le chemin d'Amour. L'étude du fr. 4 montrera, au chapitre suivant, que la division des facultés de compréhension d'un même corps est l'œuvre de la Haine. L'étude du fr. 131 révèle enfin l'effacement progressif de la persona du poète qui laisse place au discours transcendant de la Muse, inaccessible aux sens. La première partie se clôt par l'examen des métaphores du chemin, habituelles dans la poésie épique mais ici renouvelées par le lexique de l'irrigation ou de la dérivation de l'eau en canaux ( $\pi$ ópoi), liées au flot de paroles pures déversé par la Muse.

La deuxième partie se penche sur « le réemploi des formes typiques de la composition poétique archaïque » (p. 243-592). Le chapitre 5, très technique, examine comment Empédocle et Parménide refondent l'hexamètre dactylique pour l'adapter à l'expression philosophique et l'extraire de la gangue épique. L'auteur s'appuie sur une étude métrique et statistique très précise et fournie (abrègements, césures, variations de fréquence de position des mots, recensés en Annexe). Les chapitres 6 à 8 s'attachent aux trois formes typiques de composition poétique renouvelées par Empédocle, à commencer par la comparaison analogique et dysanalogique (entre œil et lanterne au fr. 84, entre œuvre zoogonique de l'Amour et création picturale au fr. 23 , entre processus de respiration et clepsydre au fr. 100). Le critère syntaxique est présenté comme « le plus pertinent » (p. 271) pour l'interprétation. Ainsi, le duel problématique au fr. 23 est compris « en-dehors des raisons doctrinales majeures » (p. 318): il n'y aurait pas deux peintres, Amour et Haine, participant simultanément à la cosmogonie, mais dualité de la série des opérations successives nécessaires pour peindre (l'Amour ne pouvant mêler que ce qui a été séparé). Le chapitre 7 montre comment la forme du catalogue de divinités (telle qu'elle apparaît aux fr. 121 à 123) est reprise des théogonies poétiques pour mieux s'en distinguer, tant par le refus de toute généalogie que de toute personnification (les anthroponymes en $-\omega$ dénotant des principes abstraits). Les répétitions (constructions annulaires et parallélismes) sont abordées au chapitre 8 , « couronnes, spirales et ritournelles $\gg$. L'auteur défend à juste titre l'idée que les compositions paratactiques, telles qu'on les trouve dans les fragments présocratiques, ne doivent pas être considérées comme « pré-rationnelles et inaptes à l'abstraction » (p. 494). Les fr. 2 et 3 fournissent un exemple d'anneau, énonçant les mêmes éléments en ordre inverse. L'auteur montre que les ritournelles (reprises entières ou partielles de vers) des fr. 17, 26, 20 et des ens. a et c du Papyrus de Strasbourg ont une valeur argumentative qui n'est ni didactique ni analogique du cycle cosmique. L'analyse sémantique et syntaxique du fr. 35 révèle une structure spiralaire imitant au sein même du fragment le déploiement diachronique des mouvements d'Amour et Haine : le contenu est à l'image de la forme, mais « ce serait aller trop loin que d'accepter l'idée qu'il y a une homologie entre le contenu de la doctrine physique et l'emploi de la ritournelle » (p. 592).

La troisième partie conclut en deux temps sur « la poésie et le projet d'Empédocle » (p. 595-705), en analysant d'abord la situation énonciative des deux poèmes («Destinataires du poème, contexte de performance et projet intellectuel »). Si, comme Xénophane et Parménide, Empédocle reprend la structure narrative des Travaux d'Hésiode, c'est pour mieux s'en dissocier : le destinataire intradiégétique du poème physique, Pausanias, figure le mortel susceptible de sortir de sa condition tragique et stylise un auditoire universel capable d'user du savoir dispensé par Empédocle. En s'adressant de même à une communauté idéale dans les Catharmes, récitées à Olympie, Empédocle se pose comme porteur d'une visée non seulement intellectuelle et éthique 
mais aussi politique, en rupture avec la poésie épicinique de son temps.

Le dernier chapitre, « la Discorde, le poète et la création poétique », réintroduit Nєîкоц, le principe antagoniste de l'Amour, que l'auteur avait délibérément écarté de l'analyse (p. 114) sauf lorsque le propos l'exigeait, car ce principe semblait ne jouer aucun rôle dans le processus de création poétique. Or de même que l'Amour a besoin de la séparation préalable par la Haine pour accomplir son œuvre, de même la création poétique présuppose un moment analytique de déconstruction de la poésie antérieure. Examinant la relation entre Haine et $\mu \alpha v i ́ n$, l'auteur propose une interprétation du fr. 115 sur l'exil du démon. Il en conclut que le poème religieux des Catharmes invite à lire le poème physique comme une compensation du crime perpétré par le démon qui s'est voué à Nعîкoৎ, en ce qu'il expose la nature véritable des choses et rend justice aux six principes divins de l'univers. La conclusion générale récapitule en quoi le projet original d'Empédocle «s'inscrit dans une triple remise en cause des constructions intellectuelles qui le précèdent : de la poésie antérieure, de la prose et de la poésie philosophique » (p. 741).

Cet ouvrage atteint son ambition, montrer l'unité indissociable entre véhicule poétique et pensée, par la mise en évidence des homologies entre forme et contenu qui en sont la manifestation épiphénoménale. On pourrait regretter que l'intertextualité poétique, de grande ampleur et profondeur, reste privilégiée par rapport à l'intertextualité philosophique, et que l'étude du « dispositif signifiant », si elle est évidemment nécessaire à la compréhension et permet d'éliminer certaines interprétations, soit parfois vue comme suffisante à la saisie du sens (par exemple quand l'auteur rejette les analyses de M. Rashed liant les fr. 84 et 100 à Perséphone / Nestis). Mais ce livre très riche et stimulant constitue à n'en pas douter une source de référence pour les études empédocléennes.

Anne-Laure THERME

Ivan Adriano Licciardi, Parmenide trádito, Parmenide tradito nel Commentario di Simplicio alla Fisica di Aristotele. Saggio introduttivo, Raccolta dei testi in greco, Traduzione e Commentario, Sankt Augustin, Academia Verlag, 2016 (Symbolon, 42), 571 p., ISBN : 978-3-89665-691-9.

Ce livre substantiel interroge la présence et le rôle de Parménide dans le Commentaire de Simplicius à la Physique d'Aristote. Il se compose d'un essai introductif, suivi d'un recueil offrant le texte grec de cinquante-trois passages du Commentaire où Simplicius se réfere à Parménide, d'une traduction italienne de ces passages judicieusement annotée, d'un commentaire analytique détaillé de ceux-ci, d'une bibliographie et de trois indices. Les passages recueillis ne sont pas de simples citations parménidiennes, mais des exégèses qui permettent de comprendre quel sens Simplicius donne aux fragments qu'il transmet. Pour le texte grec, Licciardi suit l'édition établie par Diels, avec des aménagements ponctuels justifiés dans ses notes. On attend encore à ce jour la réalisation d'une nouvelle édition critique, pour laquelle des projets sont en voie de concrétisation. L'essai introductif est ample dans sa visée et synthétique ; ses notes fournissent les références bibliographiques utiles pour approfondir les perspectives évoquées. Par ailleurs, le commentaire complète ces indications par d'autres références aussi précises qu'abondantes. Cet instrument de travail de premier plan est rendu d'autant plus maniable que les principaux résultats théoriques de l'essai sont spécifiquement articulés à la présentation des textes exposés par la suite, grâce à un précieux épilogue (p. 77-88). 